Canadian Journal of Regional Science

Revue canadienne des sciences régionales
CANADIAN JOURNAL

OF REGIONAL SCIENCE

REVUE CANADIENNE DES
SCIENCES RÉGIONALES

\title{
Note introductive « L'accès au logement et à la mobilité : nouvelles vulnérabilités métropolitaines »
}

\section{Ghislaine Deymier et Frédéric Gaschet}

Volume 43, numéro 2, 2020

Accès au logement et à la mobilité

URI : https://id.erudit.org/iderudit/1083288ar

DOI : https://doi.org/10.7202/1083288ar

Aller au sommaire du numéro

\section{Éditeur(s)}

Canadian Regional Science Association / Association canadienne des sciences régionales

\section{ISSN}

0705-4580 (imprimé)

1925-2218 (numérique)

Découvrir la revue

Citer ce document

Deymier, G. \& Gaschet, F. (2020). Note introductive « L'accès au logement et à la mobilité : nouvelles vulnérabilités métropolitaines ». Canadian Journal of Regional Science / Revue canadienne des sciences régionales, 43(2), 3-5. https://doi.org/10.7202/1083288ar 
CANADIAN JOURNAL

OF REGIONAL SCIENCE

REVUE CANADIENNE DES

SCIENCES RÉGIONALES

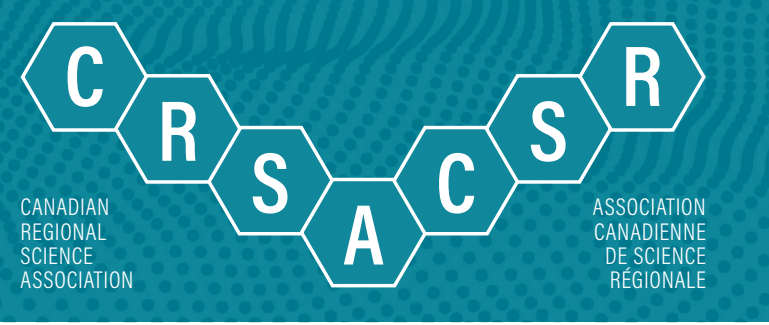

\section{NOTE INTRODUCTIVE «L'ACCÈS AU LOGEMENT ET À LA MOBILITÉ: NOUVELLES VULNÉRABILITÉS MÉTROPOLITAINES ॥}

\section{Ghislaine DEYMIER, Frédéric GASCHET}

Ghislaine DEYMIER

UMR 5319 Passages,

Université Bordeaux Montaigne
Frédéric GASCHET

UMR 5113 GREThA,

Université de Bordeaux 
Le projet de ce numéro spécial, sur le thème de la mobilité, du logement et de la vulnérabilité territoriale des ménages, a vu le jour lors des deuxièmes Rencontres Francophones Transport Mobilité (RFTM) qui se sont déroulées à Montréal du 11 au 13 juin 2019. Lors de ces rencontres, plusieurs chercheurs ont pu discuter, dans le cadre d'une session spéciale, des questions émergentes relatives aux enjeux de soutenabilité sociale des modes d'habitat et de transport liés aux formes d'urbanisation des grandes métropoles des pays francophones. Certains articles présentés dans ce numéro sont issus de cette session spéciale. D'autres se sont ajoutés par la suite, permettant d'alimenter ainsi la réflexion de façon plus large autour de ces questions.

L'ensemble des analyses menées dans ce numéro appréhendent, à des degrés divers, une problématique commune, à savoir, la difficulté de plus en plus forte des ménages de catégories moyennes et modestes, d'accéder au logement dans les zones les plus centrales des grandes agglomérations. L'une des raisons principales relève de l'accroissement considérable des prix fonciers et de l'immobilier observé dans la plupart des grandes métropoles mondiales depuis plus d'une décennie. Partout, les ménages les plus modestes et les classes moyennes sont amenés à s'éloigner largement des centres urbains pour se loger et, en particulier, pour accéder à la propriété. Ce phénomène de relégation entraîne des effets négatifs sur les mobilités des ménages concernés qui se matérialisent, notamment, par un allongement des distances et des temps de trajet et donc, par une augmentation des coûts induits par la mobilité quotidienne, d'autant que les territoires périurbains et éloignés des centres engendrent une plus grande dépendance automobile. Les ménages se trouvent ainsi confrontés à une vulnérabilité accrue face aux coûts du logement et de la mobilité. Ce phénomène fait, notamment, écho au «mouvement des gilets jaunes» qui a émergé à l'automne 2018 en France suite à la volonté du gouvernement d'augmenter la taxe Carbone. Ce mouvement a mis en lumière l'ampleur d'un phénomène que de nombreuses recherches en sociologie et économie urbaine pointaient déjà sans, toutefois, parvenir à saisir complètement le malaise profond induit par ces nouvelles fractures territoriales liées au phénomène de métropolisation et à l'accentuation des formes de ségrégation socio-spatiales (Gaschet \& Lacour, 2007). Jacques Donzelot décrivait pourtant déjà il y a quinze ans l'émergence d'une «ville à trois vitesses», rythmée par la relégation, la périurbanisation et la gentrification (Donzelot, 2004). Ces tendances ont d'ailleurs été renforcées, en France, par l'acte III de la décentralisation mis en œuvre dans les années 2010 organisant une forme «d'injonction métropolitaine» suite à la loi portant sur la Nouvelle Organisation Territoriale de la République (NOTRe) du 7 août 2015 et la loi du 27 janvier 2014 de modernisation de l'action publique et d'affirmation des métropoles (MAPTAM). Le processus de métropolisation politico-administratif ainsi mis en œuvre a, de ce fait, pu accentuer les inégalités territoriales d'accès au logement et à l'emploi, renforçant la dépendance à l'automobile de certaines catégories de ménages.

Ces mouvements soulignent ainsi la nécessité, pour penser les conditions de la transition énergétique des territoires urbains, de mieux articuler, dans l'analyse de la durabilité urbaine, la dimension environnementale et la dimension socio-économique. Trop souvent focalisées sur la seule empreinte écologique de la croissance urbaine, les approches en termes de durabilité urbaine négligent le plus souvent les enjeux de soutenabilité sociale des modes d'habitat et de transport liés aux formes d'urbanisation. La question du logement revêt pourtant une importance fondamentale dans l'appréhension des inégalités spatiales. Certains dysfonctionnements des marchés du logement ont pris une ampleur singulière, ces dernières années, tant par un accroissement conséquent du taux d'effort des ménages par rapport à son niveau de longue période que par le freinage des mobilités résidentielles, ou encore de l'incapacité à orienter la production neuve vers les zones de forte tension. Ces tensions sur l'accès au logement viennent parfois contrarier les politiques de compacité urbaine, en encourageant des comportements de localisation et des formes d'urbanisation à rebours des objectifs poursuivis. Les approches en termes de formes urbaines doivent ainsi mieux intégrer la formation des inégalités socio-spatiales.

Il convient également de mieux comprendre comment s'articulent, dans différents contextes métropolitains, les contraintes d'accès au logement et à la mobilité quotidienne, notamment pour les ménages les plus vulnérables. La mobilité quotidienne est notamment au cœur des préoccupations des territoires peu denses situés en périphérie des métropoles car elle soulève un certain nombre d'enjeux tant sur le plan environnemental, économique et social que sur l'organisation spatiale de ces territoires. Là encore, les approches existantes intègrent rarement la complexité des interactions à l'œuvre. Les analyses en termes de prix hédoniques permettent de comprendre la formation des prix du logement et leur lien avec les formes urbaines mais peinent à intégrer le rôle de l'accès à la mobilité autrement que par la prise en compte de la distance aux infrastructures et aux centralités. Inversement, la littérature sur les comportements de mobilité permet de relier les choix modaux aux formes urbaines sans pouvoir restituer pleinement les arbitrages que rendent les ménages pour gérer conjointement leurs contraintes d'accès à l'emploi, au logement et à la mobilité.

L'enjeu de ce numéro spécial est ainsi de contribuer à une meilleure compréhension des interactions complexes entre les formes urbaines, le fonctionnement des marchés du logement et les comportements de mobilité quotidienne dans différents contextes métropolitains, et à en évaluer les conséquences sur les formes et le degré de vulnérabilité des ménages.

Les articles de ce numéro montrent, ainsi, comment l'organisation spatiale des métropoles accentue les inégalités d'accès à la ville que I'on se situe dans les pays du Nord ou dans les pays en développement. Des différences importantes apparaissent, toutefois, dans les processus de relégation à l'œuvre dans les différentes métropoles étudiées, en raison de la complexité des choix de localisation des différents ménages localisés en périphérie des villes. II serait, en effet, hâtif, de considérer un processus unique de relégation des catégories moyennes et modestes, l'article de Damase Ngouma, comme celui de Ghislaine Deymier et Frédéric Gaschet, soulignant la complexité des processus de localisation des ménages, qui ne relèvent pas uniquement des arbitrages entre accessibilité, coût du logement et aménités spatiales, mis en exergue par la théorie économique urbaine, mais d'un ensemble de facteurs complexes inhérents aux caractéristiques intrinsèques des individus.

La question de l'usage de la voiture et de la dépendance automobile est au cœur des analyses menées dans l'article de Louafi Bouzouina, Caroline Bayart et Patrick Bonnel et dans celui de Nicolas Juste, Joël Meissonier et Cyprien Richer. L'automobile serait, d'un côté, un marqueur social, toujours bien ancré dans l'esprit collectif, indispensable aux populations les plus précaires souhaitant accéder à l'emploi car reléguées dans des quartiers souvent mal desservis par les transports en commun. De l'autre, elle serait moins prépondérante chez les jeunes qui auraient des aspirations modales différentes des générations précédentes. Cette désaffiliation à la culture automobile des plus jeunes n'est toutefois pas si simple, comme le montre l'étude de Louafi Bouzouina, Caroline Bayart et Patrick Bonnel sur la métropole de Lyon, qui révèle que les caractéristiques territoriales, à la fois des lieux d'emplois et de résidence, influencent fortement les besoins d'usage de la voiture et cela quelles que soient les aspirations individuelles.

Ces conclusions rejoignent celles de Nicolas Juste, Joël Meissonier et Cyprien Richer qui soulignent l'importance des formes urbaines dans l'inclusion des populations. La situation économique moyenne 
des ménages sans voiture serait ainsi meilleure dans les agglomérations de plus grande taille. Ces auteurs interrogent le «bien-fondé » de l'adage «l'automobile pour tous» qui contribuerait à perpétuer la source même des inégalités contre laquelle cette politique prétend lutter. «Considérer l'accès à l'automobile comme norme sociale indépassable dédouane de la nécessité d'interroger l'organisation même de nos villes» car "I"automobile n'a pas amélioré l'accessibilité aux lieux d'activités mais a contribué à leur dispersion ». Ainsi, les populations précaires sont condamnées à l'usage de la voiture et, par conséquent, à supporter ses coûts inhérents. Parvenir à résorber les inégalités de mobilité entre les quartiers signifie, selon les auteurs, libérer les habitants de l'obligation d'avoir recours à l'automobile et donc des coûts qu'elle engendre. L'amélioration de la situation des habitants ne pourra se réaliser qu'à la condition que les politiques de mobilité prennent en compte les spécificités territoriales. Un autre moyen d'action permettant d'améliorer l'accessibilité des catégories de population les plus précaires serait de localiser les emplois dans les secteurs desservis par des alternatives modales à la voiture comme le proposent Louafi Bouzouina, Caroline Bayart et Patrick Bonnel.

Damase Ngouma interroge, également, la façon de remédier à l'accroissement irréversible des coûts de la mobilité dans les métropoles d'Afrique subsaharienne en s'appuyant sur le cas de Brazzaville où s'observe un phénomène de "périphérisation des couches les moins aisées ». Les ménages ne disposant pas de transport efficient leur permettant d'accéder aux différentes centralités auxquelles ils aspirent sont confrontés à une vulnérabilité croissante. Ainsi, avec un «effet retard » par rapport aux métropoles du Nord, le recours au véhicule individuel motorisé s'intensifie au fur et à mesure de l'extension des villes engendrée par la pression foncière et immobilière des centres. Cette façon de concevoir les villes rend ainsi inéluctable I'usage des modes individuels motorisés afin de faciliter les interactions sociales. Le budget consacré par les ménages de ces quartiers périphériques au financement des transports devient, ainsi, excessivement élevé.

Par conséquent, le trafic s'intensifie et, comme le souligne l'article de Yves-Bryand Yao, Jean Dubé et David Lubala, cela génère des nuisances qui ont un impact non négligeable à la fois sur la santé publique, du fait des émissions de polluants et de bruit, mais également en raison des temps de déplacements accrus qui augmentent de façon exponentielle avec le recours à la voiture particulière. Ces nuisances sont d'autant plus importantes pour les ménages les plus modestes bénéficiant d'une accessibilité précaire au logement.

L'article de Yves-Bryand Yao, Jean Dubé et David Lubala révèle ainsi l'impact négatif que peut avoir un logement localisé à proximité d'une infrastructure routière à travers le développement d'une méthodologie originale d'appariement qui permet de s'émanciper des limites engendrées par l'approche hédonique pour identifier les facteurs générant des externalités négatives. L'étude proposée sur la ville de Québec montre qu'en dépit des gains d'accessibilité procurés par les infrastructures, les nuisances sont valorisées négativement dans les prix des logements jusqu'à une certaine distance, soulevant ainsi des questions d'iniquité environnementale et économique associées au développement des infrastructures routières en milieu urbain. Cette analyse doit permettre aux pays industrialisés de reconsidérer les aménagements urbains afin de limiter ces impacts négatifs et d'informer ou d'orienter les politiques publiques à venir des pays du Sud sur la nécessité de tenir compte de ces facteurs lorsqu'il faudra faciliter l'accès des espaces périphériques aux centralités.

Afin de tenir compte de toute la complexité de l'aménagement urbain et de la nécessaire prise en compte d'une approche systémique de la ville, la contribution de Nathalie Gaussier et Seghir Zerghini propose un nouvel outil de modélisation et d'aide à la décision, le modèle MUST-B. Ce modèle utilise une approche multi-agents tout en intégrant la structure classique des modèles LUTI (Land Use and Transport Interaction) afin d'appréhender la complexité des interactions entre les choix de localisation des ménages et des activités et les politiques publiques d'offre de transport et d'ouverture à l'urbanisation. La modélisation proposée permet ainsi de traiter explicitement l'interdépendance entre les choix de localisation des ménages et des entreprises, tout en prenant en compte un grand nombre de déterminants des choix de localisation résidentielle, y compris les aménités et les coûts de mobilité. La simulation réalisée sur l'agglomération de Bordeaux ouvre des perspectives prometteuses dans l'anticipation de l'impact de différents leviers d'action publique territoriale (offre et prix du transport, règle d'urbanisme...) dans une perspective d'accompagnement des politiques de transition urbaine.

La contribution de Ghislaine Deymier et Frédéric Gaschet s'inscrit, également, dans cette perspective d'approche intégrée des contraintes d'accès au logement et à la mobilité des ménages, en se focalisant sur le phénomène de la congestion urbaine. Partant du constat que les indicateurs traditionnels de congestion utilisés dans la littérature ne caractérisent que la saturation des réseaux et non les territoires de vie des ménages les plus impactés, ils proposent la mise au point d'indicateurs de congestion territorialisés fondés sur le croisement de données GPS avec les enquêtes de déplacement. Ces indicateurs permettent d'analyser l'impact de la congestion urbaine sur les différents types de territoires d'une métropole, en prenant en compte le profil socio-économique des ménages et le coût d'accès au logement sur ces territoires. L'application proposée sur l'aire métropolitaine de Bordeaux montre que la congestion frappe de façon disproportionnée les territoires périurbains à revenu modeste, confirmant que la relégation de populations à faible revenu dans des territoires éloignés des centres d'emplois en raison des coûts du logement les expose à un niveau de congestion très important, qui renforce leur vulnérabilité.

L'ensemble de ces contributions démontre tout l'intérêt de développer des travaux de recherche à l'interface du logement, des transports et de la vulnérabilité des ménages et des territoires. Ils invitent à mieux articuler, dans les politiques de transition urbaine, les questions environnementales et les questions sociales. L'effet des principaux instruments des politiques de transition urbaine doit être apprécié en fonction des contextes territoriaux et des politiques sociales menées à différentes échelles sur ces territoires.

Les articles rassemblés dans ce numéro spécial soulignent, in fine, la fécondité d'une approche centrée sur la vulnérabilité des ménages et des territoires, élaborée de façon multidimensionnelle, à travers la prise en compte des contraintes de logement, de mobilité et de dépenses énergétiques.

\section{RÉFÉRENCES BIBLIOGRAPHIQUES}

Donzelot, J. (2004). La ville à trois vitesse: relégation, périurbanisation, gentrification, Esprit, 3-4: 14-39.

Gaschet F. \& Lacour C. (2007). Métropolisation et ségrégation socio-spatiale, Bordeaux, Presses universitaires de Bordeaux. 